\title{
Stage IIA2 Cervical Cancer FIGO 2018
}

National Cancer Institute

\section{Source}

National Cancer Institute. Stage IIA2 Cervical Cancer FIGO 2018. NCI Thesaurus. Code C162237.

Cervical carcinoma limited to the upper two-thirds of the vag ina without parametrial involvement, equal or more than $4 \mathrm{~cm}$ in greatest dimension. (FIGO 2018) 